\title{
Suprasellar Benign Chondroma: A Case Report and Literature Review
}

\author{
Satoshi Nakanowatari, ${ }^{1}$ Katsumi Sakata, ${ }^{1}$ Ryohei Miyazaki, ${ }^{1}$ Takashi Kawasaki, ${ }^{1}$ and Hiroshi Manaka
}

\begin{abstract}
Intracranial chondroma is a rare benign tumor comprising only $0.2 \%$ of all intracranial tumors. A 27 -year-old woman presented with visual dysfunction and headache. Brain computed tomography and magnetic resonance imaging revealed a suprasellar mass lesion with a calcified component. Gross total removal was achieved via a basal interhemispheric approach. Postoperatively, visual function improved to the normal range, and no recurrence was evident 4 years later. Histopathological examination confirmed the diagnosis of benign chondroma. Preoperative differentiation of chondromas from chordomas is clinically important, because of the different treatment and prognostic implications. The only effective treatment for chondroma is total surgical removal. We present a case of gross total resection of a suprasellar chondroma with reference to the literature.
\end{abstract}

Keywords: chondroma, suprasellar tumor, basal interhemispheric approach

\section{Introduction}

Chondromas usually arise from embryonic chondrocytic cell remnants in the small bones of the hands and feet, and consist of mature hyaline cartilaginous tissue. ${ }^{1,2)}$ Synchondrosis joints are found between the occipital and sphenoid bones, and between the petrous portion of the temporal bone and jugular process of the occipital bone. Intracranial chondromas frequently occur at the skull base of the parasellar region, clivus or cerebello-pontine angle. ${ }^{3-9)}$ Intracranial chondromas are rare benign tumors comprising $0.2 \%$ of all intracranial tumors. ${ }^{5,6)}$ In the clinical field, a chondroma resembles a chordoma and a chondrosarcoma in terms of radiographic images and pathological features. ${ }^{5,10)}$ Preoperative differentiation between chondromas and other tumors is thus often difficult. ${ }^{5,11)}$ However, differential diagnosis is also quite important, because the prognoses can be markedly different. ${ }^{5,12,13)}$ Here, we report the complete surgical resection of a suprasellar chondroma via the basal interhemispheric approach and present a review of the literature.

\footnotetext{
${ }^{1}$ Department of Neurosurgery, Yokohama City University Medical Center, Yokohama, Kanagawa, Japan
}

Received: June 13, 2019; Accepted: October 7, 2019 Online March 24, 2020

Copyright $₫ 2020$ by The Japan Neurosurgical Society This work is licensed under a Creative Commons AttributionNonCommercial-NoDerivatives International License.

\section{Case Report}

A 27-year-old woman presented with a 1-year history of visual disturbance. At 26 years old, she had developed nepheropsia. One year later, she had experienced slight headache, and magnetic resonance imaging (MRI) revealed a suprasellar tumor. She was then referred to our hospital. On examination, visual acuity was motus manus in the right eye and temporal hemianopsia in the left eye. Other neurological findings were intact. Pituitary function was normal.

\section{Imaging Studies}

Computed tomography (CT) showed a suprasellar mass lesion with a slightly calcified component (Fig. 1a). The mass lesion was located in the midline of the suprasellar region. Bone imaging revealed osteolytic changes and bony destruction of the posterior clinoid process (Fig. 1b). MRI showed the suprasellar mass lesion was hypointense on T1-weighted images (Fig. 2a), hyperintense on T2-weighted images (Fig. $2 \mathrm{~b}$ ) and a heterogeneous-honeycomb-enhanced lesion on gadolinium contrast-enhanced MRI (Figs. 2c and 2d). The tumor was attached to the diaphragma sellae and elevated the optic chiasm (Figs. 2a and 2b).

\section{Operation and Postoperative Course}

Because this tumor containing a calcified component was located behind the optic chiasm, we selected a basal interhemispheric approach to obtain a wide operating field and remove the calcified hard tissue radically. After dissection of the interhemispheric fissure, a dark-yellowish tumor was encountered. The tumor contained a large calcified component and a smaller gelatinous component. The calcified lesion had markedly elevated the optic nerve, however could be dissected from the nerve via a wide prechiasmatic window. The pituitary stalk was identified behind the tumor capsule. Gross total removal was achieved. The tumor origin was considered to be the dura of the posterior clinoid process, and some part of the dural attachment was left in place. The postoperative course was uneventful including the pituitary function. Visual symptoms improved to the normal range. MRI at 4 years postoperatively showed no recurrence (Fig. 3).

\section{Histopathological Findings}

Histopathological examination revealed lobular proliferation of chondrocytes with homogeneous small nuclei and lacunar structures indicating chondroid differentiation against a cartilaginous matrix background (Fig. 4). Tumor cells were arranged without tight cell-to-cell adhesion of 
the epithelial type and without physaliphorous cells associated with the chordoma. Cells did not show nuclei with increased chromatin and two small nuclei accompanied by the chondrosarcoma. No nuclear atypia or pleomorphism, or evidence of mitosis were seen., ${ }^{2,310)}$ Immunochemistry disclosed positive findings for vimentin and negative findings for cytokeratin and epithelial membrane antigen. ${ }^{2)}$ Based on these findings, the pathological diagnosis was a benign chondroma.

\section{Discussion}

Intracranial chondromas were first reported by Hirschfield in $1851 .{ }^{14)}$ Approximately $15 \%$ of intracranial chondromas arise supratentorially from the dura mater, brain parenchyma, ventricle or parafalcine locations. ${ }^{4,6-9)}$ Skull-base chondromas are usually divided into suprasellar, parasellar and clival types, ${ }^{1,3)}$ which may arise from the residual primordial cartilaginous cranium, such as at the spheno-petrosal, petro-occipital and spheno-occipital synchondroses. ${ }^{1,3,4)}$ Most skull-base chondromas are therefore located in the paramedian area. ${ }^{6,7)}$

In general, suprasellar tumors in adults are relatively common, such as giant pituitary adenoma, meningioma and craniopharyngioma. A midline suprasellar chondroma is a rare benign tumor among the primary intracranial tumors, with an estimated incidence rate of $0.2 \%$, and is difficult to
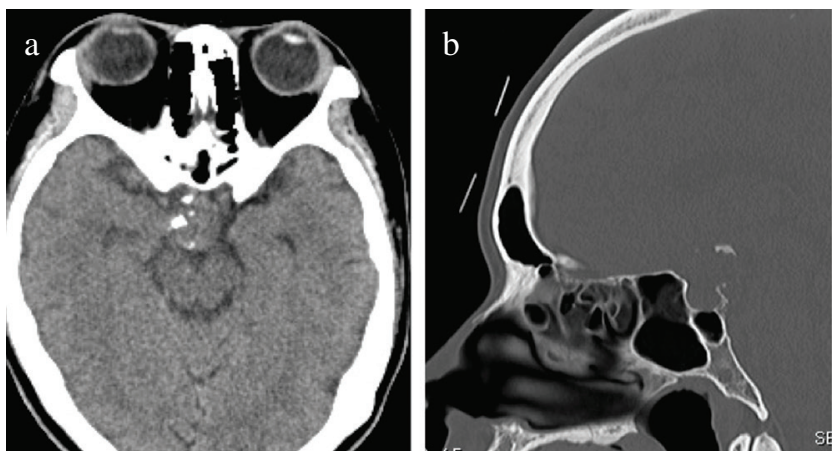

Fig. 1 Computed tomography (CT). (a) Axial CT shows a suprasellar, isodense mass lesion with a calcified component. (b) Bone window sagittal CT reveals a bone-density component in the suprasellar portion. preoperatively distinguish from other suprasellar tumors, such as chordomas, chondrosarcomas and craniopharyngiomas. ${ }^{2,5,10,11)} \mathrm{CT}$ reveals a lobularly circumscribed mass with regional calcification accompanied by erosion and destruction of the surrounding bone structures. ${ }^{6,715)}$ Coronal CT with a bone window shows multiple small circular and curvilinear calcifications occurring in the myxochondroid matrix. Calcifications are recognized as irregular shapes in $60 \%$, with
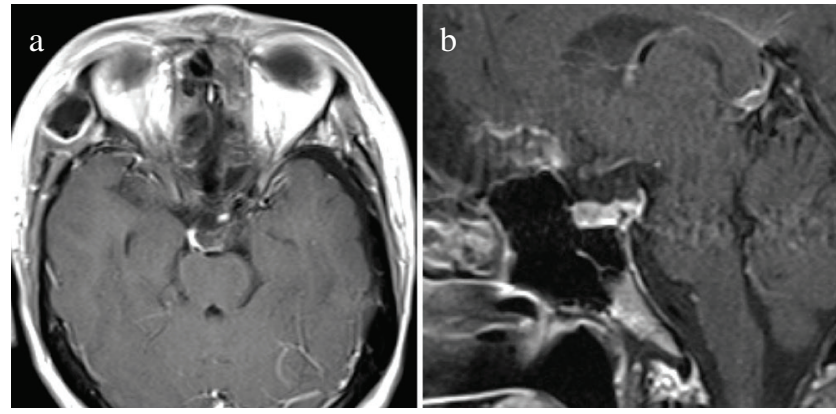

Fig. 3 Postoperative magnetic resonance imaging (MRI) shows successful removal. (a) Axial contrast-enhanced T1-weighted MRI. (b) Sagittal contrast-enhanced T1-weighted MRI.

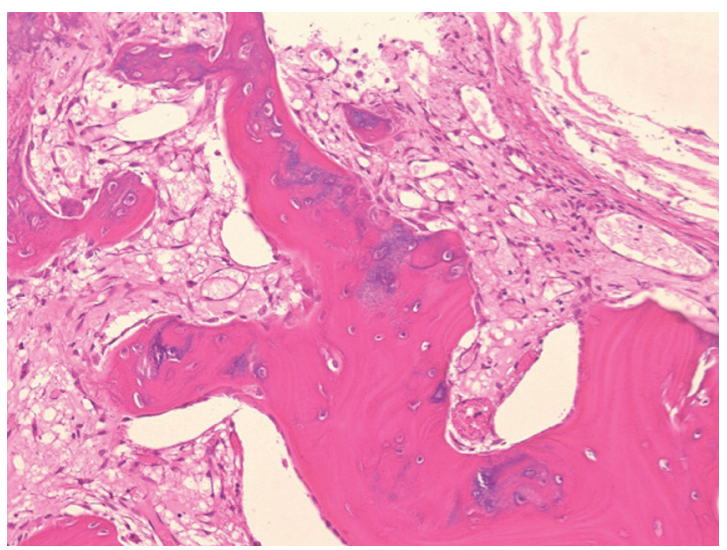

Fig. 4 Histopathological findings (hematoxylin and eosin $\times 100$ ). The tumor against the myxoid matrix background comprises cellular hyaline cartilage with irregular lobules, spindle cells and chondrocytes with homogeneous small nuclei.
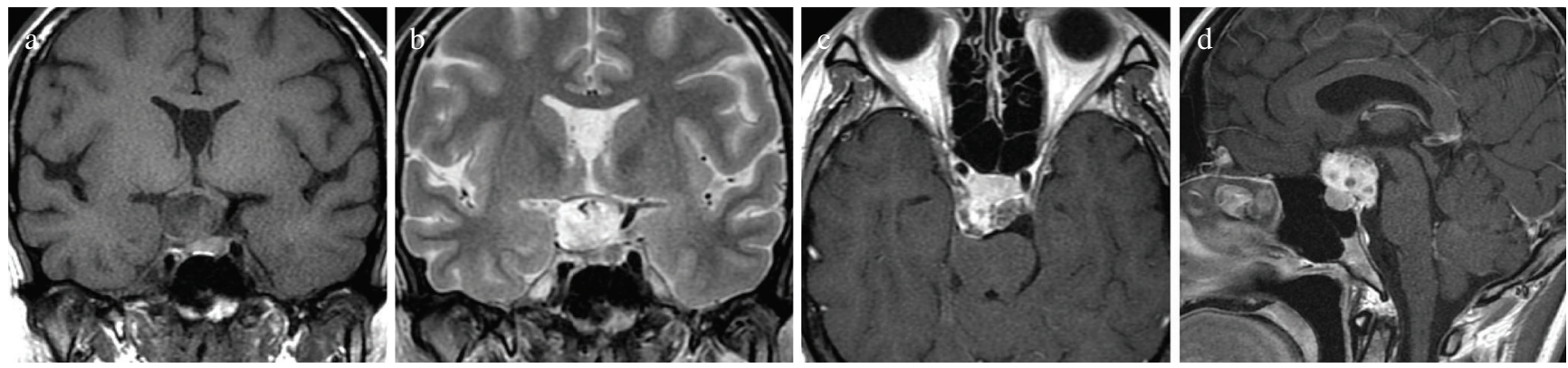

Fig. 2 Magnetic resonance imaging (MRI). (a) Coronal T1-weighted MRI shows a suprasellar hypointense mass lesion elevating the optic chiasm. (b) Coronal T2-weighted MRI reveals hyperintensity. (c) Axial contrast-enhanced T1-weighted MRI shows a heterogeneously enhanced mass compressing the posterior part of bilateral cavernous sinuses. (d) Sagittal contrast-enhanced T1-weighted MRI shows a suprasellar, heterogeneously enhanced mass extending to the interpeduncular cistern and elevating the optic chiasm. 
local bone destruction in $40 \% .^{2,6,7)}$ Chordomas have been reported to comprise $40-50 \%$ of lesions showing calcifications and $50-60 \%$ of those with bone destruction. ${ }^{5,10)}$ Similar calcified findings are also seen in patients with meningiomas or craniopharyngiomas. Bone destruction is often identified in both chondromas and chordomas, but less frequently than with chondromas. ${ }^{6)}$ Clinical symptoms in patients with suprasellar chondromas are always associated with visual dysfunction. ${ }^{1,15,16)}$ Differential diagnosis between skull-base chordomas and low-grade chondrosarcomas could be quite important. Chordomas also locally invade to the midline and parasellar skull base with similar clinical and radiological characteristics, given their origin from embryonic notochord remnants in the midline. ${ }^{5,10,11)}$ Pathological diagnosis is also very important, because the treatment strategies and prognoses differ. ${ }^{12,13)}$

In patients with suprasellar benign chondromas, the only way to achieve a cure is to remove the tumor totally. No recurrence is expected following complete resection of the tumor, and the long-term prognosis is acceptable. ${ }^{14,17-19)}$ Past reports on surgical operations have described various approaches, including the trans-sphenoidal approach, transbasal interhemispheric approach, extra-dural temporopolar approach, and anterior trans-petrosal approach. ${ }^{12,14,19)}$ In this case, we chose the basal interhemispheric approach to obtain a wide operating field surrounding the prechiasmatic window and to remove the calcified component behind the optic chiasm, although endoscopic transnasal surgery has recently been developed as an alternative surgical approach. The progression-free survival rate has been reported as $75 \%$ at 5 years and the overall survival rate as $93 \% .{ }^{19)}$ In addition, visual dysfunction could be expected to improve after surgical resection. On the other hand, the 5-year overall survival rate for a patient with a chordoma is only $50-60 \%$, because total removal of the tumor is difficult and tumor recurrence is frequent. ${ }^{5,10)}$ Radiation therapy is ineffective and malignant transformation to chondrosarcoma has been reported after partial removal. ${ }^{2,19)}$ Radiation therapy is therefore not recommended.

In conclusion, a suprasellar chondroma is a rare benign intracranial neoplasm. Differential diagnosis is quite important because of the possibility of clinically similar suprasellar neoplasms with different prognoses. Surgical resection is the treatment of choice. In our case, gross total removal was achieved via a basal interhemispheric approach, followed by dramatic improvements in visual symptoms.

\section{Conflicts of Interest Disclosure}

The authors have no conflicts of interest to declare.

\section{References}

1) de Divitiis E, Spaziante R, Cirillo S, Stella L, Donzelli R: Primary sellar chondromas. Surg Neurol 11: 229-232, 1979

2) Patel A, Munthali L, Bodi I: Giant cystic intracranial chondroma of the falx with review of literature. Neuropathology 29: 315-317, 2009

3) Falconer MA, Bailey IC, Duchen LW: Surgical treatment of chordoma and chondroma of the skull base. J Neurosurg 29: 261-275, 1968

4) Lacerte D, Gagné F, Copty M: Intracranial chondroma. Report of two cases and review of the literature. Can J Neurol Sci 23: 132-137, 1996

5) Almefty K, Pravdenkova S, Colli BO, Al-Mefty O, Gokden M: Chordoma and chondrosarcoma: similar, but quite different, skull base tumors. Cancer 110: 2457-2467, 2007

6) Brownlee RD, Sevick RJ, Rewcastle NB, Tranmer BI: Intracranial chondroma. AJNR Am J Neuroradiol 18: 889-893, 1997

7) De Coene B, Gilliard C, Grandin C, Nisolle JF, Trigaux JP, Lahdou JB: Unusual location of an intracranial chondroma. AJNR Am J Neuroradiol 18: 573-575, 1997

8) Kurt E, Beute GN, Sluzewski M, van Rooij WJ, Teepen JL: Giant chondroma of the falx. Case report and review of the literature. J Neurosurg 85: 1161-1164, 1996

9) Nakayama M, Nagayama T, Hirano H, Oyoshi T, Kuratsu J: Giant chondroma arising from the dura mater of the convexity. Case report and review of the literature. J Neurosurg 94: 331-334, 2001

10) Rosenberg AE, Nielsen GP, Keel SB, et al.: Chondrosarcoma of the base of the skull: a clinicopathologic study of 200 cases with emphasis on its distinction from chordoma. Am J Surg Pathol 23: 1370-1378, 1999

11) Gay E, Sekhar LN, Rubinstein E, et al.: Chordomas and chondrosarcomas of the cranial base: results and follow-up of 60 patients. Neurosurgery 36: 887-896; discussion 896-897, 1995

12) Samii A, Gerganov VM, Herold C, et al.: Chordomas of the skull base: surgical management and outcome. J Neurosurg 107: 319-324, 2007

13) Sen C, Triana AI, Berglind N, Godbold J, Shrivastava RK: Clival chordomas: clinical management, results, and complications in 71 patients. J Neurosurg 113: 1059-1071, 2010

14) Heo J, Cho SJ: A case of giant skull base chondroma. Brain Tumor Res Treat 2: 92-95, 2014

15) Reuter K, Weber AL: Parasellar chondrosarcoma in a patient with Ollier's disease. Neuroradiology 22: 151-154, 1981

16) Terasaka S, Sawamura Y, Abe H: Surgical removal of a cavernous sinus chondroma. Surg Neurol 48: 153-159, 1997

17) Acampora S, Troisi F, Fusco G, Del Gaizo S: Voluminous intracranial chondroma. Surg Neurol 18: 254-257, 1982

18) Mapstone TB, Wongmongkolrit T, Roessman U, Ratcheson RA: Intradural chondroma: a case report and review of the literature. $\mathrm{Neu}$ rosurgery 12: 111-114, 1983

19) Weng JC, Li D, Li H, et al.: Surgical management and outcomes of intracranial chondromas: a single-center case series of 66 patients. World Neurosurg 108: 264-277, 2017 